\title{
Mining relationships between food groups and eating time slots and diabetes status in adults from UK NDNS RP
}

\section{Abstract}

The timing of energy/nutrient intake has been previously shown to be associated with obesity and diabetes (Almoosawi et al, 2019) and recently derived diurnal patterns of energy/carbohydrate intake suggested the potential interplay of circadian biology and social behaviour contributing to obesity (Palla\&Almoosawi, 2019) but didn't describe the relationship between food groups and the time when they are eaten, nor on how such relationships vary by type 2 diabetes status.

To assess such relationships in a representative sample of the British adult population (19-64 ys old), we have cross-classified 60 food groups and 7 time-slots (6-9am, 9-12,12-2pm,2-5pm, 5-8pm, 8-10pm, 10-6am) as recorded in the National Diet and Nutrition Survey Rolling Programme (2008-2014) and analysed the derived frequency by Correspondence Analysis (CA) of food entries, producing biplots separately for the foods included in the food healthiness score tertiles, for all adults combined and separately by diabetes status (self-reported diabetics with $\mathrm{HbAlc}>6.5 \%$ and glucose $<6.1 \mathrm{mmol} / \mathrm{L}$, prediabetics with $\mathrm{HbA} 1 \mathrm{c}<6.5 \%$ and glucose comprised between 6.1 and 7 , undiagnosed diabetics with $\mathrm{HbAlc}>6.5$ but not self-reported and non diabetics). Where numbers allowed, the odds ratio estimate was derived of consuming unhealthy food groups later in the day compared to earlier in the day, by logistic regression.

The general CA biplots (representing about $80 \%$ or more of the total system inertia) pointed at a very strong association between evening/night and beer, spirits and a strong association between evening/night and ice-cream, sugar confectionary, chocolate, biscuits, crisps. The odds ratio (95\% CIs) of consuming the listed foods at night evening rather than earlier were respectively 4.37 (CI:3.755.10), 7.78 (CI:6.11-9.91),1.63 (CI:1.34-1.98), 1.59 (1.24-2.03), 2.06 (CI:1.84-2.31), 1.53(CI:1.39-1.68), 1.26 (CI:1.11-1.42). The stratified biplots were mostly consistent with the findings for general CA biplots except for puddings which additionally appeared strongly associated with evening time for prediabetics and undiagnosed diabetics but not for diabetics and non-diabetics. The limited number of diabetics $(n=84,2.3 \%)$, undiagnosed diabetics $(n=41,1.1 \%)$ and prediabetics $(n=63,1.7 \%)$ and high diabetes status missingness $(n$ $=1445,39.5 \%)$ prevented from applying regression analyses within subgroups.

Assessing the relationships between less healthy foods and timing of eating is a first step towards identifying specific public health targets for behaviour change, which include alcoholic beverages and foods rich in added sugars and saturated fats. The greater likelihood of eating puddings in the evening might partly explain the undiagnosed/prediabetic state. The survey cross-sectional nature warrants further investigations by longitudinal cohort studies.

\section{Conflict of Interest}

There is no conflict of interest 